\title{
CTL Model Checking Based on Forward State Traversal
}

\author{
Hiroaki Iwashita Tsuneo Nakata Fumiyasu Hirose \\ Fujitsu Laboratories Ltd. \\ 1-1 Kamikodanaka 4-chome, Nakahara-ku, Kawasaki 211-88, Japan \\ hiroaki@flab.fujitsu.co.jp
}

\begin{abstract}
We present a CTL model checking algorithm based mainly on forward state traversal, which can check many realistic CTL properties without doing backward state traversal. This algorithm is effective in many situations where backward state traversal is more expensive than forward state traversal. We combine it with BDD-based state traversal techniques using partitioned transition relations. Experimental results show that our method can verify actual CTL properties of large industrial models which cannot be handled by conventional model checkers.
\end{abstract}

\section{Introduction}

Formal verification techniques are starting to become standard industrial hardware verification methods, since people know that design complexity continues to grow and that they cannot keep up using only conventional verification methods, such as logic simulation. For sequential systems, one of the most popular and feasible approaches to formal verification is CTL model checking [1, 2, 3]. It is a method for verifying that a sequential system satisfies a property where the system is modeled as a finite state machine (FSM) and the property is expressed in a temporal logic called Computation Tree Logic (CTL) [1].

Rapid progress has been made in model checking techniques since binary decision diagrams (BDDs) [4] were incorporated into them $[5,6]$. It has been shown that these techniques, called symbolic model checking, can potentially verify large FSMs with more than $10^{20}$ states. The effectiveness of symbolic model checking, however, heavily depends on the model's structure and BDD variable ordering. In practice, symbolic model checking of a complicated system can only be accomplished by a few experts who can fine tune the model based on their knowledge of both the target system and the symbolic model checker.

Some techniques using partitioned transition relations have been proposed to verify larger FSMs [7, 8]. Memory usage is reduced by making the partition size smaller, in general. We often observe, however, CPU time for backward state traversal becomes much larger when we make the partition size smaller. We cannot always find a good partitioning for backward state traversal that satisfies both memory and time limitations. On the other hand, we also observe that CPU time for forward state traversal is not affected by the partition size so much. We can use partitioned transition relations composed of individual latch transition functions to reduce memories, without great loss of execution speed.

In the symbolic model checking paradigm, CTL formulas have been evaluated with backward state traversal [2]. We describe, in this paper, an algorithm to accomplish CTL model checking in the opposite direction. It is effective in many situations where backward state traversal is more expensive than forward state traversal. After preliminaries in Section 2, Section 3 characterizes forward state traversal and backward state traversal using partitioned transition relations. Section 4 presents our new procedure for evaluating CTL properties with forward state traversal. We report the results in Section 5.

\section{Preliminaries}

\subsection{Transition relations}

An FSM is a 6-tuple, $\left(S, I, O, \delta, \lambda, s_{0}\right)$, where $S$ is the set of states, $I$ is the set of input values, $O$ is the set of output values, $\delta: S \times I \rightarrow S$ is the next state function, $\lambda: S \times I \rightarrow$ $O$ is the output function, and $s_{0} \in S$ is the initial state. In what follows, let $B=\{0,1\}$.

The transition relation of an FSM is the function $T$ : $S \times I \times S \rightarrow B ; T(x, i, y)=1$ iff $y=\delta(x, i)$. In the basic symbolic technique, a single BDD is built for representing the transition relation $T$. For a large FSM, however, we often fail to construct the BDD for $T$ because of a BDD size explosion. Partitioned transition relations [8] are popular representation to reduce the BDD size. When the state is expressed by a vector of $n$ Boolean state variables (latches) and the transition function of the $k$-th latch is given by $\delta_{k}(\vec{x}, \vec{i})$, we can make a conjunctive partitioned transition relation as follows:

$$
\begin{aligned}
T(\vec{x}, \vec{i}, \vec{y}) & =T_{1}\left(\vec{x}, \vec{i}, y_{1}\right) \wedge \ldots \wedge T_{n}\left(\vec{x}, \vec{i}, y_{n}\right) \\
T_{k}\left(\vec{x}, \vec{i}, y_{k}\right) & =\left(y_{k} \equiv \delta_{k}(\vec{x}, \vec{i})\right)
\end{aligned}
$$


Latch transition relations $T_{1}, \ldots, T_{n}$ are represented by $n$ BDDs, which are much smaller than the BDD for $T$ in general.

\subsection{Images and pre-images}

Given an $\operatorname{FSM}\left(S, I, O, \delta, \lambda, s_{0}\right)$ and a set of states $A \subseteq S$, the image of $A$ is defined to be the set of states $\{y \mid \exists x \in$ $A, \exists i \in I, y=\delta(x, i)\}$, and the pre-image of $A$ is defined by the set of states $\{x \mid \exists y \in A, \exists i \in I, y=\delta(x, i)\}$.

A set of states $A \subseteq S$ can be represented by a characteristic function $\chi_{A}: S \rightarrow B ; \chi_{A}(x)=1$ iff $x \in A$. We use a notation $\mathcal{L}(f)$ to express the set of states represented by function $f$, in this paper. The image and the pre-image of $\mathcal{L}(f)$ are calculated by following symbolic operations:

$$
\begin{aligned}
& \operatorname{Img}(f)(\vec{y}) \\
& \quad=\exists \vec{x} \cdot \exists \vec{i} \cdot[T(\vec{x}, \vec{i}, \vec{y}) \wedge f(\vec{x})] \\
& \quad=\exists \vec{x} \cdot \exists \vec{i} \cdot\left[T_{1}\left(\vec{x}, \vec{i}, y_{1}\right) \wedge \ldots \wedge T_{n}\left(\vec{x}, \vec{i}, y_{n}\right) \wedge f(\vec{x})\right] \\
& \operatorname{Pre}(f)(\vec{x}) \\
& \quad=\exists \vec{i} \cdot \exists \vec{y} \cdot[T(\vec{x}, \vec{i}, \vec{y}) \wedge f(\vec{y})] \\
& \quad=\exists \vec{i} \cdot \exists \vec{y} \cdot\left[T_{1}\left(\vec{x}, \vec{i}, y_{1}\right) \wedge \ldots \wedge T_{n}\left(\vec{x}, \vec{i}, y_{n}\right) \wedge f(\vec{y})\right]
\end{aligned}
$$

The image and the pre-image of $\mathcal{L}(f)$ are $\mathcal{L}(\operatorname{Img}(f))$ and $\mathcal{L}($ Pre $(f))$ respectively. Operations $\operatorname{Img}(f)$ and Pre $(f)$ are similar if $T$ is given by a single BDD. When we use conjunctive partitioned transition relations, early existential quantification can be done while calculating the conjunction of all BDDs [7, 8]. Efficiency of the calculation strongly depends on the order in which the BDDs are processed. Strategies to find effective orders for $\operatorname{Img}(f)$ and $\operatorname{Pre}(f)$ seem to be different, because those expressions have different forms.

\subsection{CTL model checking}

Model checking is the process of determining whether a model (FSM) satisfies its requirements (properties). A temporal logic CTL [1] is commonly used to express properties about an FSM. CTL formulas are composed of atomic propositions with usual logical operators and following temporal operators:

- EX $f(\mathbf{A X} f)$ which means that $f$ holds at some (every) successor state of the current state.

- EF $f$ (AF $f$ ) which means that for some (every) state transition path, there exists a state on the path at which $f$ holds.

- EG $f$ (AG $f$ ) which means that for some (every) state transition path, $f$ keeps holding forever on the path.
- $\mathbf{E}[g \mathbf{U} f](\mathbf{A}[g \mathbf{U} f])$ which means that for some (every) state transition path, there exists a state on the path at which $f$ holds, and $g$ holds at all the preceding states.

A CTL property is expressed by a notation like " $M, s=$ $f$." It means that the CTL formula $f$ is true in state $s$ of model $M$. It is also written simply as " $s \models f$ " where the model is not ambiguous.

CTL formula $f$ can be interpreted as a set of states $\mathcal{L}(f)=\{s|s|=f\}$. EX $f$ is then the same operation as computing the pre-image of $\mathcal{L}(f)$ :

$$
\mathbf{E X} f=\operatorname{Pre}(f)
$$

$\mathbf{E}[g \mathbf{U} f]$ and $\mathbf{E G} f$ can be characterized by the least and greatest fix-point computation as follows:

$$
\begin{aligned}
\mathbf{E}[g \mathbf{U} f] & =\mathbf{l f p} Z[f \vee(g \wedge \mathbf{E X} Z)] \\
\mathbf{E G} f & =\mathbf{g f p} Z[f \wedge \mathbf{E X} Z]
\end{aligned}
$$

The remaining operators are given by following rules:

$$
\begin{aligned}
\mathbf{E F} f & =\mathbf{E}[\operatorname{true} \mathbf{U} f] \\
\mathbf{A X} f & =\neg \mathbf{E X} \neg f \\
\mathbf{A F} f & =\neg \mathbf{E} \mathbf{G} \neg f \\
\mathbf{A G} f & =\neg \mathbf{E F} \neg f \\
\mathbf{A}[g \mathbf{U} f] & =\neg(\mathbf{E}[\neg f \mathbf{U} \neg g \wedge \neg f] \vee \mathbf{E G} \neg f)
\end{aligned}
$$

A fairness constraint is a condition representing fair state transition paths in which we are interested. CTL model checking under fairness constraints is performed by restricting state transition paths along which each fairness constraint holds infinitely often. Fairness constraints are given by a set of CTL formulas $C$. CTL formula EG $f$ under fairness constraints in $C$ is computed as follows [2]:

$$
\mathbf{E}_{C} \mathbf{G} f=\operatorname{gfp} Z\left[f \wedge \mathbf{E X} \bigwedge_{c \in C} \mathbf{E}[Z \mathbf{U} Z \wedge c]\right]
$$

The set of states that are the start of some fair path under fairness constraints in $C$ is given by $\mathcal{L}\left(\mathbf{E}_{C} \mathbf{G}\right.$ true). Once $\mathbf{E}_{C} \mathbf{G}$ true is evaluated, $\mathbf{E X} f$ and $\mathbf{E}[g \mathbf{U} f]$ under fairness constraints in $C$ can be computed simply as follows:

$$
\begin{aligned}
\mathbf{E}_{C} \mathbf{X} f & =\mathbf{E X}\left(f \wedge \mathbf{E}_{C} \mathbf{G} \text { true }\right) \\
\mathbf{E}_{C}[g \mathbf{U} f] & =\mathbf{E}\left[g \mathbf{U}\left(f \wedge \mathbf{E}_{C} \mathbf{G} \text { true }\right)\right]
\end{aligned}
$$

\section{Forward versus backward traversal}

Conventional CTL model checkers evaluate CTL formulas with repeated pre-image computation, backward state traversal. Properties $s_{0}=\mathbf{E F} f$ and $s_{0}=\mathbf{A G} f$ are also 


\begin{tabular}{|l||rlr|l|}
\hline \multicolumn{1}{|c|}{ Model } & \#Latches & \#States & Depth & \multicolumn{1}{c|}{ Description } \\
\hline \hline gigamax & 16 & $1.2 \times 10^{2}$ & 8 & An example in VIS distribution (originated in SMV). \\
atm_sw & 54 & $2.0 \times 10^{5}$ & 126 & Abstracted processor core for an ATM-switch [9]. \\
dh_1 & 46 & $4.0 \times 10^{3}$ & 21 & Cache coherency protocol description focused on a multiprocessor BUS. \\
dh_2 & 66 & $7.9 \times 10^{6}$ & 17 & Cache coherency protocol description focused on processor interaction. \\
vpp & 101 & $2.4 \times 10^{11}$ & 18 & Abstracted VLIW microprocessor pipeline. \\
pipe_s & 35 & $2.9 \times 10^{8}$ & 11 & Abstracted superscalar microprocessor pipeline; a simple version. \\
pipe_d & 73 & $5.7 \times 10^{17}$ & 11 & Abstracted superscalar microprocessor pipeline; a complicated version. \\
\hline
\end{tabular}

Table 1: Benchmark examples

\begin{tabular}{|l||r|r|r||r|r|r|}
\hline \multicolumn{1}{|c||}{ Model } & \multicolumn{3}{c||}{ Complete TR } & \multicolumn{3}{c|}{ Partitioned TR } \\
\cline { 2 - 7 } & \#Nodes & Image & Pre-image & \#Nodes & Image & Pre-image \\
\hline \hline gigamax & $0.5 \mathrm{~K}$ & 0.0 & 0.0 & $1 \mathrm{~K}$ & 0.3 & 0.0 \\
atm_Sw & $1302 \mathrm{~K}$ & 8.9 & 21.8 & $1 \mathrm{~K}$ & 10.9 & 202.5 \\
dh_1 & $3 \mathrm{~K}$ & 0.0 & 0.8 & $1 \mathrm{~K}$ & 0.3 & 1.3 \\
dh_2 & $100 \mathrm{~K}$ & 13.8 & 75.4 & $35 \mathrm{~K}$ & 46.8 & $>10000$ \\
vpp & N/A & N/A & N/A & $60 \mathrm{~K}$ & 3.0 & 4135.1 \\
pipe_s & $322 \mathrm{~K}$ & 10.7 & 14.8 & $3 \mathrm{~K}$ & 0.6 & 387.9 \\
pipe_d & N/A & N/A & N/A & $348 \mathrm{~K}$ & 200.4 & $>20000$ \\
\hline
\end{tabular}

Table 2: CPU seconds per image or pre-image computation

known to be verified by comparing $\mathcal{L}(f)$ and the reachable states. Reachable states are enumerated with repeated image computation, forward state traversal.

Performance of the computation is very sensitive to BDD variable ordering. It is difficult to find a good variable order automatically, and ordinary users cannot always find it manually. When we use conjunctive partitioned transition relations, the performance is also sensitive to the order in which the BDDs are processed. In our experience of industrial hardware verification, however, image computation with partitioned transition relation works relatively fine even if the FSM is very large and the ordering is not tuned so much.

Table 1 summarizes seven models that we use as benchmark examples in this paper. Excepting gigamax, the models and those properties are actual ones that we have prepared for verifying real hardware products. For each benchmark, we tried conventional model checking procedure that checks reachability with forward state traversal and evaluates CTL formulas with backward state traversal. We used both a complete transition relation represented by a single BDD and a conjunctive partitioned transition relation represented by a set of BDDs for latch transition relations.

Table 2 shows average CPU time per image or preimage computation during each model checking process. Total numbers of BDD nodes for complete/partitioned transition relations are also shown in the table. Complete transition relations for models vpp and pipe_d could not be made because of BDD size explosions. Pre-image computation with partitioned transition relation for models $d h \_2$ and pipe_d exceeded the CPU time limit of 24 hours. We should not compare image computation time and pre-image computation time directly, because they are solving different problems. The results, however, show that the time ratio of image computation and pre-image computation with a partitioned transition relation is huge, while that with a complete transition relation is relatively small.

\section{Forward CTL model checking}

As described in Section 3, there is a large CPU time difference between image computation and pre-image computation especially when a conjunctive partitioned transition relation is used. We should traverse state space forward when we can choose the direction. Although CTL model checking has been based on backward state traversal, we propose a new method for checking CTL properties using forward state traversal in this section.

\subsection{Rewriting property notations}

A CTL property is given as a notation like " $s_{0} \mid=f$." Conventional model checking procedure matches the notation: it evaluates CTL formula $f$ with backward state traversal, and then checks if it holds at state $s_{0}$. We rewrite the notation for the purpose of matching it with our method. We translate the CTL property into a problem of comparing a formula with the constant false. Let $s_{0}$ be a state of the 
FSM, $p_{0}$ the characteristic function of $\left\{s_{0}\right\}$, and $f$ an arbitrary CTL formula. Formula $p_{0}$ is true only at state $s_{0}$, and formula $f$ is true at state $s_{0}$ iff $s_{0}=f$ holds. Therefore, the " $=$ " notation can be rewritten as follows:

$$
\begin{aligned}
& s_{0}=f \quad \Longleftrightarrow p_{0} \wedge f \neq \text { false } \\
& s_{0} \models f \quad \Longleftrightarrow p_{0} \wedge \neg f=\text { false }
\end{aligned}
$$

Some model checkers support models with multiple initial states, while " $=$ " represents relation between a single state and a CTL formula. Given a set of initial states $S_{0}$, we believe that interpretation of some extended notation like " $S_{0} \mid=f$ " is ambiguous. It should be written as " $\exists s \in S_{0}, s=f$ " or " $\forall s \in S_{0}, s=f$ ". They can be rewritten as " $p_{0} \wedge f \neq$ false" and " $p_{0} \wedge \neg f=$ false" respectively where $p_{0}$ is the characteristic function of $S_{0}$.

\subsection{Forward EX evaluation}

Let $p$ and $f$ be formulas. We can replace an outermost $\mathbf{E X}$ evaluation with image computation as follows:

$$
p \wedge \mathbf{E X} f \neq \text { false } \Longleftrightarrow \operatorname{Img}(p) \wedge f \neq \text { false }
$$

Proof: Assume $p \wedge \mathbf{E X} f$ holds at state $s$. Then $p$ holds at $s$ and $f$ holds at some successor state of $s$, say $t$. $\operatorname{Img}(p)$ holds at $t$ since $\operatorname{Img}(p)$ holds at any successor state of $s \in$ $\mathcal{L}(p)$. Thus, $\operatorname{Img}(p) \wedge f$ holds at $t$. Conversely, assume $\operatorname{Img}(p) \wedge f$ holds at state $t$. Then $f$ holds at $t$ and $t$ is a successor state of some state $s \in \mathcal{L}(p)$. EX $f$ holds at $s$ since $t \in \mathcal{L}(f)$ is a successor state of $s$. Thus, $p \wedge \mathbf{E X} f$ holds at state $s$.

Notice that we have removed an operator $\mathbf{E X}$ from $f$. Using equation (3) again or using one of the equations described later, it is possible to continue conversion of a backward traversal operator in $f$ into a forward traversal operator.

\subsection{Forward EU evaluation}

We define a state enumeration procedure under constraints given by two formulas $p$ and $q$ :

$$
F_{w d U n t i l}(p, q)=\operatorname{lfp} Z[p \vee \operatorname{Img}(Z \wedge q)]
$$

An element of $\mathcal{L}\left(F_{w d U n t i l}(p, q)\right)$ is a state $t$ such that there exists a path through $t$ from some state at which $p$ holds, and $q$ holds at all states before $t$ on the path.

Using the FwdUntil () operator, we can replace an outermost EU evaluation as follows:

$$
\begin{aligned}
p \wedge \mathbf{E}[q \mathbf{U} f] & \neq \text { false } \\
& \Longleftrightarrow F w d U n t i l(p, q) \wedge f \neq \text { false }
\end{aligned}
$$

Proof: Assume $p \wedge \mathbf{E}[q \mathbf{U} f]$ holds at state $s$. Then both $p$ and $\mathbf{E}[q \mathbf{U} f]$ hold at $s$. It means that there exists a path from $s$ through some state $t \in \mathcal{L}(f)$, and $q$ holds at all states before $t$. Thus, FwdUntil $(p, q) \wedge f$ holds at $t$. Conversely, assume $F_{w d U n t i l}(p, q) \wedge f$ holds at state $t$. Then both $F w d U n t i l(p, q)$ and $f$ holds at $t$. There exists a path through $t$ from some state $s \in \mathcal{L}(p)$, and $q$ holds at all states before $t$. Thus, $p \wedge \mathbf{E}[q \mathbf{U} f]$ holds at $s$.

Now the operator $\mathbf{E U}$ have been removed from $f$. Thus, we have a chance again to convert a backward traversal operator in $f$ into a forward traversal operator, as in equation (3).

\subsection{Forward EG evaluation}

We define an operator like EG, except that pre-image computation is replaced by image computation:

$$
E H(p)=\operatorname{gfp} Z[p \wedge \operatorname{Img}(Z)]
$$

$E H(p)$ is used to check whether there exists a state transition cycle in $\mathcal{L}(p)$. $\mathcal{L}(E H(p))$ is the subset of $\mathcal{L}(p)$ such that every state is reachable from a cycle through states only in $\mathcal{L}(p)$. We also define simple composite operators:

$$
\begin{aligned}
\operatorname{Reachable}(p, q) & =F w d \operatorname{Until}(p, q) \wedge q \\
F w d \operatorname{Global}(p, q) & =\operatorname{EH}(\operatorname{Reachable}(p, q))
\end{aligned}
$$

Reachable $(p, q)$ computes the subset of $\mathcal{L}(q)$ whose elements can be reached from $\mathcal{L}(p \wedge q)$ through states only in $\mathcal{L}(q)$. FwdGlobal $(p, q)$ checks whether there exists a state transition cycle in $\mathcal{L}(q)$ that is reachable from $\mathcal{L}(p \wedge q)$ through states only in $\mathcal{L}(q)$.

Using the FwdGlobal () operator, we can replace an outermost EG evaluation as follows:

$$
\begin{aligned}
p \wedge \mathbf{E G} q & \neq \text { false } \\
\Longleftrightarrow & \Longleftrightarrow \text { FwdGlobal }(p, q) \neq \text { false }
\end{aligned}
$$

Proof: Assume $p \wedge \mathbf{E G} q$ holds at state $s$. Then both $p$ and EG $q$ holds at $s$. It means that for some path from $s, q$ keeps holding forever on the path. In other words, there exists a cycle in $\mathcal{L}(q)$ and it is reachable from $s$ through states only in $\mathcal{L}(q)$. $\mathcal{L}($ Reachable $(p, q))$ includes the cycle, since it includes all the states reachable from $s \in \mathcal{L}(p \wedge q)$ through states only in $\mathcal{L}(q)$. Thus, $\operatorname{EH}($ Reachable $(p, q)) \neq$ false. Conversely, assume $E H($ Reachable $(p, q)) \neq$ false. There exists a cycle in $\mathcal{L}($ Reachable $(p, q))$. It means that the cycle is in $\mathcal{L}(q)$ and is reachable from some state $s \in \mathcal{L}(p)$ through states only in $\mathcal{L}(q)$. Thus, $p \wedge$ EG $q$ holds at $s$.

\subsection{Forward fair EG evaluation}

We also introduce fairness constraints into forward CTL evaluation. The key is exactly like ordinary fair CTL evaluation, a procedure to find fair cycles. The procedure that 
compute $E H(p)$ under fairness constraints $C$ is given as follows:

$\operatorname{FairEH}(p)=\operatorname{gfp} Z\left[p \wedge \operatorname{Img}\left(\bigwedge_{c \in C} \operatorname{Reachable}(c, Z)\right)\right]$

We then modify the FwdGlobal() operator to handle fairness constraints using FairEH():

$$
\text { FwdFairGlobal }(p, q)=\operatorname{FairEH}(\operatorname{Reachable}(p, q))
$$

Using the FwdFairGlobal() operator, we can replace an outermost EG evaluation under fairness constraints as follows:

$$
\begin{aligned}
p \wedge \mathbf{E}_{C} \mathbf{G} q & \neq \text { false } \\
& \Longleftrightarrow \text { FwdFairGlobal }(p, q) \neq \text { false }
\end{aligned}
$$

It is clear from the fact that both sides are the modified version of equation (5) that restrict paths under the same constraints.

\subsection{The conversion procedure}

Using conversion rules (3), (4), (5), and (6), we can replace $\mathbf{E X}, \mathbf{E U}, \mathbf{E G}$, and $\mathbf{E}_{C} \mathbf{G}$ with forward traversal operators. An original property notation should be rewritten using either positive form (1) or negative form (2) so that the formula matches one of the rules. The problem of comparing a disjunctive expression with the constant false, such as " $f \vee g \neq$ false", can be divided into sub-problems, such as " $f \neq$ false" and " $g \neq$ false". We can check each term separately, and if one or more terms are not the constant false, the entire expression is not the constant false. We do not need to convert all CTL temporal operators into forward traversal operators. Remaining operators can be evaluated in usual manner, with backward state traversal. Hence, all CTL formulas can be handled with our method. The conversion procedure is shown below:

1. Rewrite the CTL formula only in temporal operators $\mathbf{E X}, \mathbf{E U}, \mathbf{E G}$, and $\mathbf{E}_{C} \mathbf{G}$.

2. Translate " $="$ notation into an expression comparing a formula with the constant false, using equation (1) or (2).

3. Arrange outermost logical operations in disjunctive form, and divide the problem into a set of subproblems comparing each product term with the constant false.

4. For each sub-problem, convert a backward operator to a forward operator using one of equations (3), (4), (5), and (6), if applicable.
5. For each newly updated sub-problems, call the procedure recursively from step 3 .

Although steps 2 and 4 have choice, it is easy to find good conversion for actual CTL properties. Many properties that we examined can be fully converted to forward state traversal problems, as shown in the next section.

Example Here is an example of converting one of the most common properties, "whenever a request is made, acknowledgment will return in the future," where req means the request, ack means the acknowledgment, $s_{0}$ is the initial state, and $p_{0}$ is the characteristic function of $\left\{s_{0}\right\}$ :

$$
\begin{aligned}
s_{0} & \models \mathbf{A G}(\text { req } \rightarrow \mathbf{A F} \text { ack }) \\
& \Longleftrightarrow s_{0} \models \neg \mathbf{E}[\text { true } \mathbf{U}(\text { req } \wedge \mathbf{E G} \neg \text { ack })] \\
& \Longleftrightarrow p_{0} \wedge \mathbf{E}[\text { true } \mathbf{U}(\text { req } \wedge \mathbf{E G} \neg \text { ack })]=\text { false } \\
& \Longleftrightarrow \text { FwdUntil }\left(p_{0}, \text { true }\right) \wedge(\text { req } \wedge \mathbf{E G} \neg \text { ack })=\text { false } \\
& \Longleftrightarrow\left(\text { FwdUntil }\left(p_{0}, \text { true }\right) \wedge \text { req }\right) \wedge \mathbf{E G} \neg \text { ack }=\text { false } \\
& \Longleftrightarrow \text { FwdGlobal }\left(\left(\text { FwdUntil }\left(p_{0}, \text { true }\right) \wedge \text { req }\right), \neg a c k\right)=\text { false } .
\end{aligned}
$$

\section{Experimental results}

\subsection{Applicability to actual CTL properties}

Our method becomes effective when many temporal operators in a CTL formula are converted into our forward traversal operators. We investigated examples in two existing symbolic model checkers SMV [2] and VIS [10], and also examined our own property examples.

We found that $90 \%$ of the properties can be rewritten using only the forward traversal operators: 18 properties out of 20 in the SMV examples, 47 properties out of 55 in the VIS examples, and all of our 13 properties. The rest of the properties are classified into three types:

$$
\begin{aligned}
& s_{0} \models \mathbf{A G E F} a \\
& \Longleftrightarrow F w d \text { Until }\left(p_{0}, \text { true }\right) \wedge \neg \mathbf{E F} a=\text { false } \\
& s_{0} \models \mathbf{A G}(a \rightarrow \mathbf{E G} b) \\
& \Longleftrightarrow F w d \text { Until }\left(p_{0}, \text { true }\right) \wedge a \wedge \neg \mathbf{E G} b=\text { false } \\
& s_{0} \models \mathbf{A G}((a \rightarrow \mathbf{E X} b) \wedge(b \rightarrow \mathbf{E X} a)) \\
& \Longleftrightarrow\left\{\begin{array}{l}
F w d U n t i l\left(p_{0}, \text { true }\right) \wedge a \wedge \neg \mathbf{E X} b=\text { false } \\
F w d \text { Until }\left(p_{0}, \text { true }\right) \wedge b \wedge \neg \mathbf{E X} a=\text { false }
\end{array}\right.
\end{aligned}
$$

where $a$ and $b$ are atomic propositions. Both forward and backward traversal are used to check these properties. They are, in fact, the same as conventional methods that can unfold only outermost AG operators.

\subsection{Performance of CTL model checking}

We measured execution speed and memory usage of our model checker, named BINGO. We also executed SMV 


\begin{tabular}{|c|c|c|c|c|}
\hline \multirow[b]{2}{*}{ Model } & \multirow[b]{2}{*}{ SMV } & \multirow[b]{2}{*}{ VIS } & \multicolumn{2}{|c|}{ BINGO } \\
\hline & & & Bwd & Fwd \\
\hline \multirow{2}{*}{ gigamax } & $3.6 \mathrm{sec}$ & $21.3 \mathrm{sec}$ & $9.3 \mathrm{sec}$ & $9.1 \mathrm{sec}$ \\
\hline & $1.6 \mathrm{MB}$ & $4.9 \mathrm{MB}$ & $3.7 \mathrm{MB}$ & $3.7 \mathrm{MB}$ \\
\hline \multirow{2}{*}{ atm_sw } & $1734 \mathrm{sec}$ & \multirow{2}{*}{ space } & $9447 \mathrm{sec}$ & $1158 \mathrm{sec}$ \\
\hline & 8.3MB & & $27.1 \mathrm{MB}$ & $23.1 \mathrm{MB}$ \\
\hline \multirow{2}{*}{$d h \_1$} & \multirow{2}{*}{ - } & $138.7 \mathrm{sec}$ & $29.6 \mathrm{sec}$ & $20.3 \mathrm{sec}$ \\
\hline & & $6.8 \mathrm{MB}$ & $3.7 \mathrm{MB}$ & $3.8 \mathrm{MB}$ \\
\hline \multirow{2}{*}{ dh_2 } & \multirow{2}{*}{ - } & \multirow{2}{*}{ space } & \multirow{2}{*}{ time } & $1357 \mathrm{sec}$ \\
\hline & & & & $31.5 \mathrm{MB}$ \\
\hline \multirow{2}{*}{ vpp } & \multirow{2}{*}{ time* } & \multirow{2}{*}{ space } & $45569 \mathrm{sec}$ & $223.3 \mathrm{sec}$ \\
\hline & & & $17.5 \mathrm{MB}$ & $13.2 \mathrm{MB}$ \\
\hline \multirow{2}{*}{ pipe_s } & $23715 \mathrm{sec}$ & $51.7 \mathrm{sec}$ & $12429 \mathrm{sec}$ & $64.6 \mathrm{sec}$ \\
\hline & $60.2 \mathrm{MB}$ & $6.1 \mathrm{MB}$ & $5.5 \mathrm{MB}$ & $4.9 \mathrm{MB}$ \\
\hline \multirow{2}{*}{ pipe_d } & \multirow{2}{*}{ - } & \multirow{2}{*}{ space } & \multirow{2}{*}{ time } & $8979 \mathrm{sec}$ \\
\hline & & & & $249.7 \mathrm{MB}$ \\
\hline
\end{tabular}

'time' exceeded 24 hours in CPU time.

'space' exceeded 300 megabytes of memory.

' $*$ failed while building a transition relation.

\section{Table 3: Performance of CTL model checking}

(version 2.2.4, released in 1994) and VIS (version 1.0, released in 1995) as references. BINGO makes a conjunctive partitioned transition relation composed of individual latch transition functions. Nondeterministic relations are translated into deterministic functions by adding unconstrained pseudo inputs. SMV makes a complete transition relation [2]. VIS makes a conjunctive partitioned transition relation by clustering several transition functions together [10].

We tried all models shown in Table 1 on BINGO and VIS. We also tried gigamax, atm_sw, vpp, and pipe_s on SMV. The gigamax description for SMV has a slightly different construction from that for others. Excepting gigamax, we ran the experiments for all model checkers with the same BDD variable order, which is defined statically according to the original model description. We also tested dynamic variable reordering on VIS; essential improvement could not be observed, however. Transition relation clustering in VIS is left unchanged from its default. The results are shown in Table 3. CPU times and memories were measured on a $50 \mathrm{MHz}$ SPARCstation 10 . We set a CPU time limit of 24 hours and a memory limit of 300 megabytes. BINGO is executed in two modes: the conventional model checking mode that unfolds only an outermost AG or EF operator (labeled "Bwd"), and the forward traversal mode that uses the algorithm presented in this paper (labeled "Fwd").

The results indicate that a fully partitioned transition relation and forward CTL model checking made a successful combination in BINGO. Some large models cannot be verified without using fully partitioned transition relations.
Execution speed of forward CTL model checking is the fastest or nearly the fastest of the methods. It is remarkable that "Fwd" is extremely faster than "Bwd" in the large models that require fully partitioned transition relations.

\section{Conclusion}

In this paper, we have presented techniques for verifying CTL properties using forward state traversal. They can be mixed with conventional backward CTL evaluation techniques, and are applicable to arbitrary CTL properties. Most realistic properties can be converted to use only forward state traversal, while the class of such properties is still uncertain. When our method is combined with BDDbased state traversal techniques using partitioned transition relations, it becomes a very practical method for verifying large FSMs.

\section{References}

[1] E. M. Clarke, E. A. Emerson, and A. P. Sistla, "Automatic Verification of Finite-State Concurrent Systems Using Temporal Logic Specifications," in ACM Transitions on Programming Languages and Systems, 8(2), pp. 244-263, 1986.

[2] K. L. McMillan, Symbolic Model Checking, Kluwer Academic Publishers, 1993.

[3] E. M. Clarke, O. Grumberg, K. L. McMillan, and X. Zhao, "Efficient Generation of Counterexamples and Witness in Symbolic Model Checking," in Proc. 32nd DAC, pp. 427432, 1995.

[4] R. E. Bryant, "Graph Based Algorithm for Boolean Function Manipulation," in IEEE Transactions on Computers, C-35(8), pp. 677-691, 1986.

[5] J. R. Burch, E. M. Clarke, K. L. McMillan, and D. L. Dill, "Sequential Circuit Verification Using Symbolic Model Checking," Proc. 27th DAC, pp. 46-51, 1990.

[6] O. Coudert and J. C. Madre, "A Unified Framework for the Formal Verification of Sequential Circuits," in Proc. ICCAD90, pp. 126-129, 1990.

[7] H. J. Touati, H. Savoj, B. Lin, R. K. Brayton, and A. Sangiovanni-Vincentelli, "Implicit State Enumeration of Finite State Machines using BDD's," in Proc. ICCAD-90, pp. 130-133, 1990.

[8] J. R. Burch, E. M. Clarke, and D. E. Long, "Representing Circuits More Efficiently in Symbolic Model Checking," in Proc. 28th DAC, pp. 403-407, 1991.

[9] B. Chen, M. Yamazaki, and M. Fujita, "Bug Identification of a Real Chip Design by Symbolic Model Checking," in Proc. European Design and Test Conference, pp. 132-136, 1994.

[10] The VIS Group, "VIS: A System for Verification and Synthesis," in Proc. Conference on Computer Aided Verification, 1996. 